\title{
Surgical Wound Management in Dogs using an Improved Stable Chlorine Dioxide Antiseptic Solution.
}

\author{
Chapnick $\mathrm{A}^{1}$ and Wilkins $\mathrm{RJ}^{* 2}$ \\ ${ }^{1}$ Veterinary Surgeon, Veterinary Healthcare Associates, 3025 Dundee Rd, Winter Haven FL 33884 \\ ${ }^{2}$ Consultant Veterinary Clinical Pathologist, R\&B Management Inc, 1810 Columbia Drive, Englewood FL 34223
}

${ }^{*}$ Corresponding author: Wilkins RJ, BVSc, DVP, DACVP, Consultant Veterinary Clinical Pathologist, R\&B Management Inc, 1810 Columbia Drive, Englewood FL 34223, E-mail: rejuvenatn@aol.com

Citation: Chapnick A, Wilkins RJ (2014) Surgical Wound Management in Dogs using an Improved Stable Chlorine Dioxide Antiseptic Solution. J Vet Sci Anim Husb 2(1): 103. doi: 10.15744/2348-9790.1.403

Received Date: December 12, 2013 Accepted Date: February 21, 2014 Published Date: February 24, 2014

\begin{abstract}
Three surgical case reports are presented to demonstrate the clinical efficacy of using an improved aqueous solution of chlorine dioxide complex $(160 \mathrm{ppm})$ as a topical antiseptic in the post operative management of serious wounds in dogs. In vitro studies are included to demonstrate the antiseptic properties of this new chlorine dioxide complex.
\end{abstract}

Keywords: Chlorine dioxide; Antiseptic; Antimicrobial; Wound management

Abbreviations

$\mathrm{ClO}_{2}$-Chlorine dioxide; $\mathrm{Cl}_{2}$-Chlorine; PPM-Parts Per Million; SPP-Species; TEM-Transmission Electron Micrograph

\section{Introduction}

With the emergence of antibiotic resistant bacteria, more virulent strains, and new pathogens, the need to find more effective antimicrobial agents for the treatment of wounds in veterinary medicine is imperative. One such agent is chlorine dioxide. A new proprietary formulation of chlorine dioxide, a stable complex in aqueous solution at $160 \mathrm{ppm}$. (Ciderm ${ }^{\star} \mathrm{SP}^{\star}$ ) has recently been introduced for veterinary use.

This formulation has been demonstrated, in in-vitro laboratory studies, to kill bacteria in one minute, and is equally microbicidal against viruses, yeast and fungi. Three clinical case examples are presented representing over 30 cases treated successfully over a one year period using this chlorine dioxide complex solution as part of the post operative wound management program. The results demonstrated that this chlorine dioxide complex solution is effective in the management of difficult wounds, both in treating and preventing infection, while preserving viable tissue.

\section{Main Body}

\section{Case Study 1}

A 10 year old, female spayed, Whippet, presented with a subcutaneous mass caudal to the left shoulder. The mass had been previously biopsied and diagnosed as a soft tissue sarcoma. The lesion was surgically excised and closed with an advancement subdermal plexus skin flap. Concurrently the dog was being treated for immune mediated disease with cyclosporine and prednisone and was immune compromised.

Eleven days after the resection the dog represented with partial necrosis of the skin flap (Figure1). The wound was surgically debrided and treatment with chlorine dioxide was initiated. The wound was flushed with saline and then sprayed with chlorine dioxide complex solution* and allowed to dry on the wound. This was repeated twice daily for 5 days, then once daily until the wound closed by second intention over the following 3 weeks (Figure 2). At 2 month follow-up examination, healing and hair regrowth was complete (Figure 3 ).

* Ciderm ${ }^{\circledast}$ SP Topical Antiseptic Solution: Frontier Pharmaceutical Melville NY 11747 (USA) 


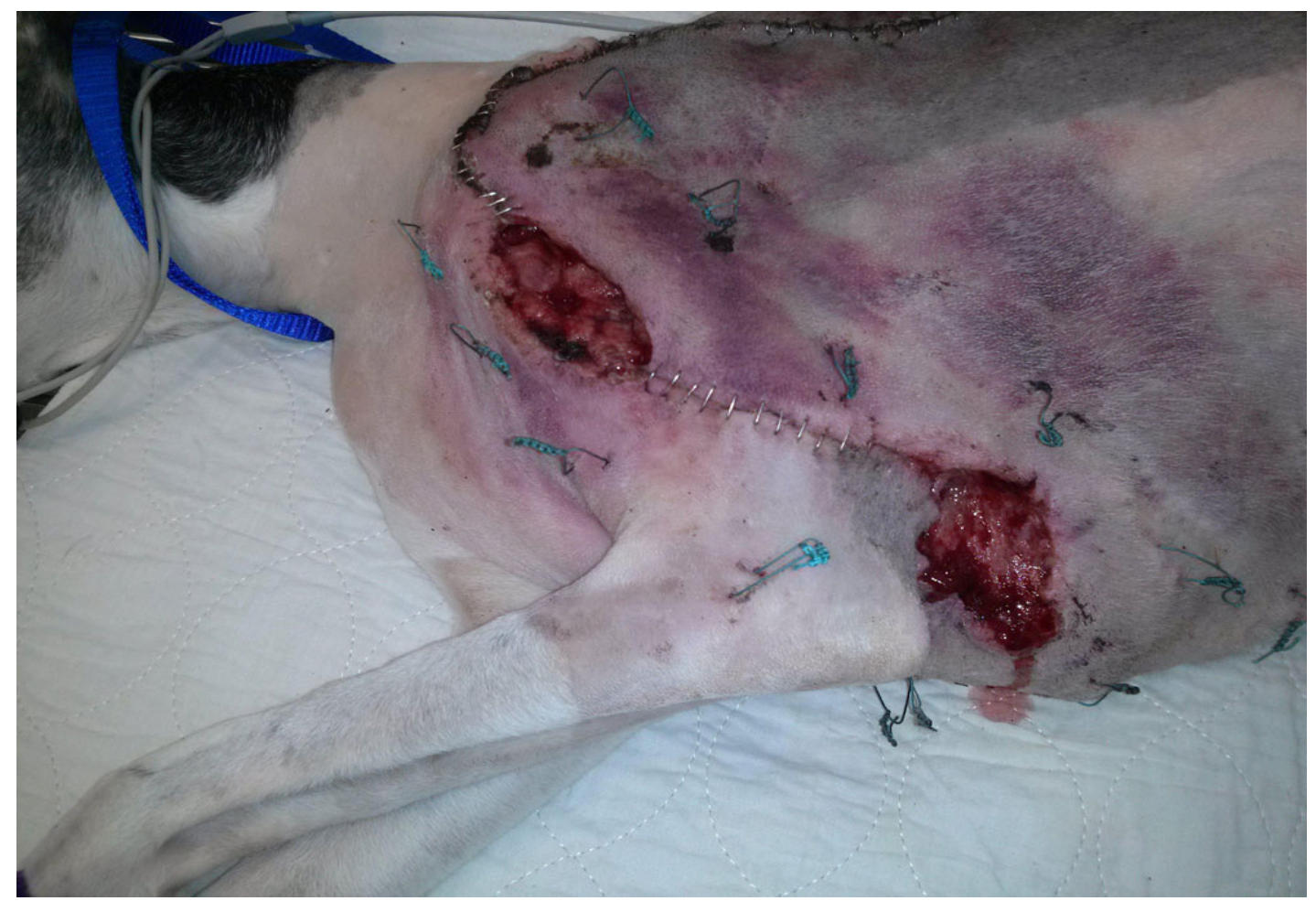

Figure 1: Dehisced Wound, inflamed and secondarily infected, 11 days post initial surgery. The lesion was irrigated and treated topically with chlorine dioxide complex antiseptic solution*

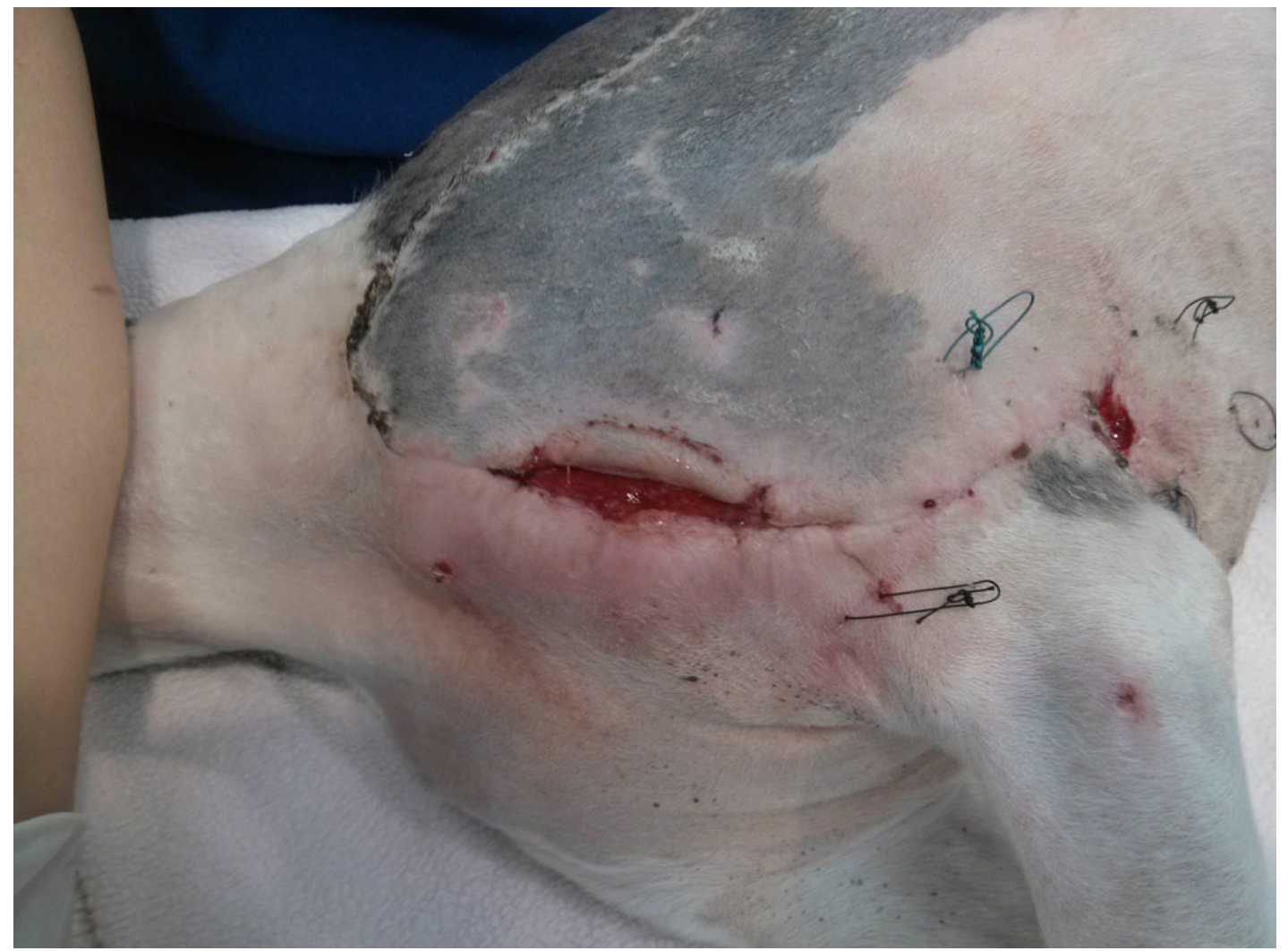

Figure 2: Wound status 10 days post initiation of chlorine dioxide solution irrigation 


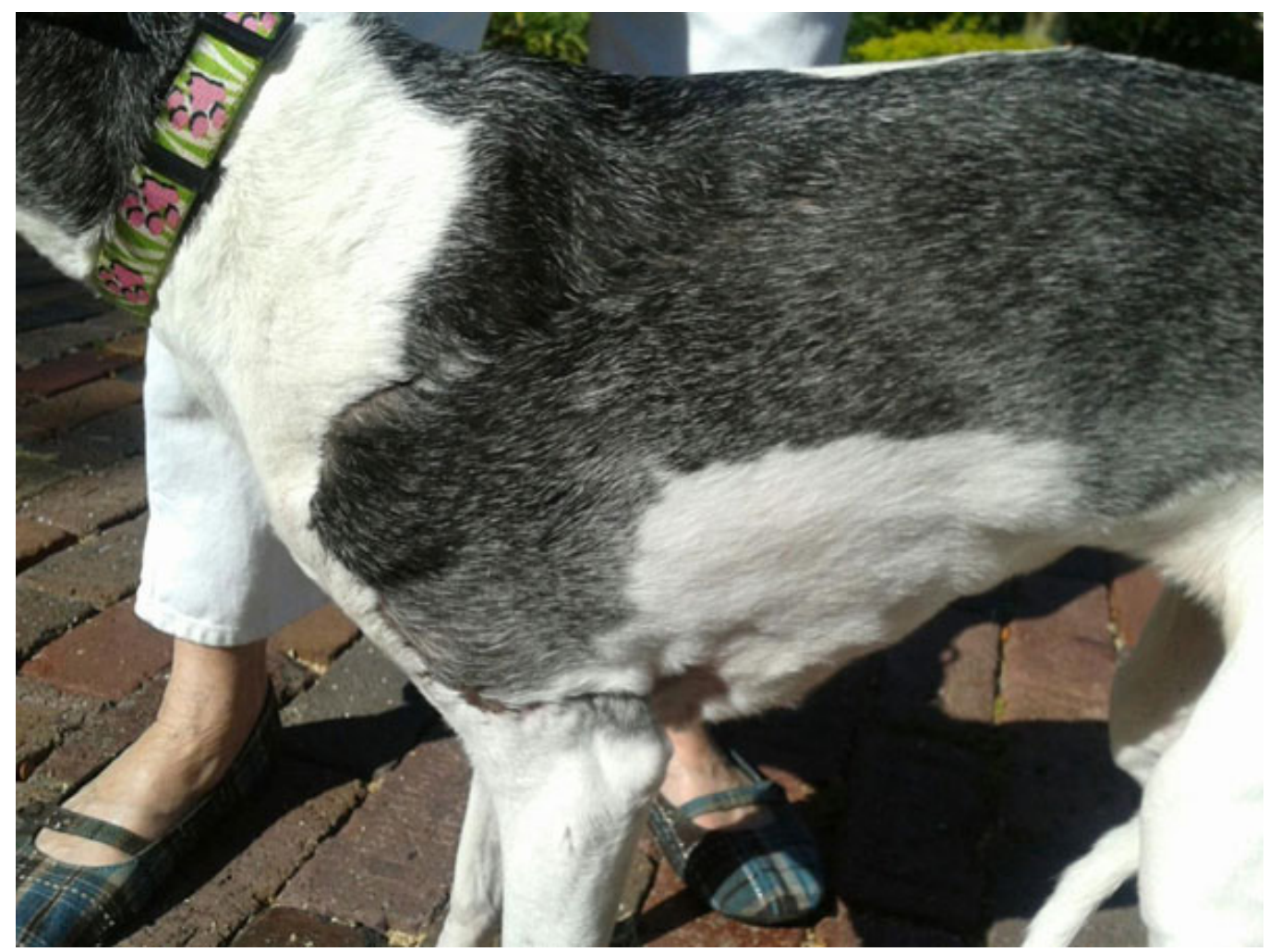

Figure 3: Healed wound without further surgical closure. Photo taken 2 months post injury

\section{Case Study 2}

An 8 year old female spayed Greyhound/Husky mix was missing for several days, returning with degloving wounds on both forelimbs. The wounds were managed by debridement and cleansing with chlorine dioxide antiseptic solution daily. The injured right carpal joint was surgically stabilized with transarticular external fixation. The exposed granulated wound (Figure 4) was grafted 21 days after the initial injury with a full thickness meshed skin graft (Figure 5). Wound care with chlorine dioxide solution was continued as the wounds healed and contracted over a 2 month period with no secondary infection developing (Figures 6-9).

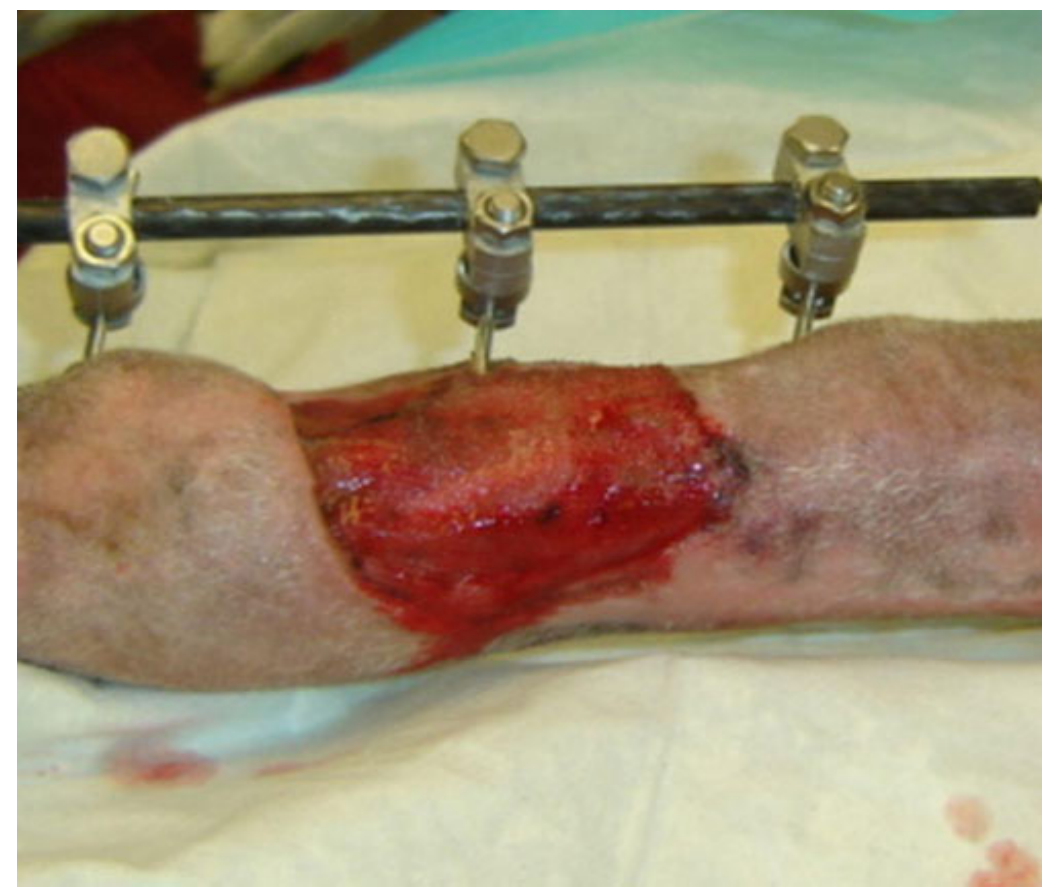

Figure 4: Wound 21 days post injury. Ready for graft 


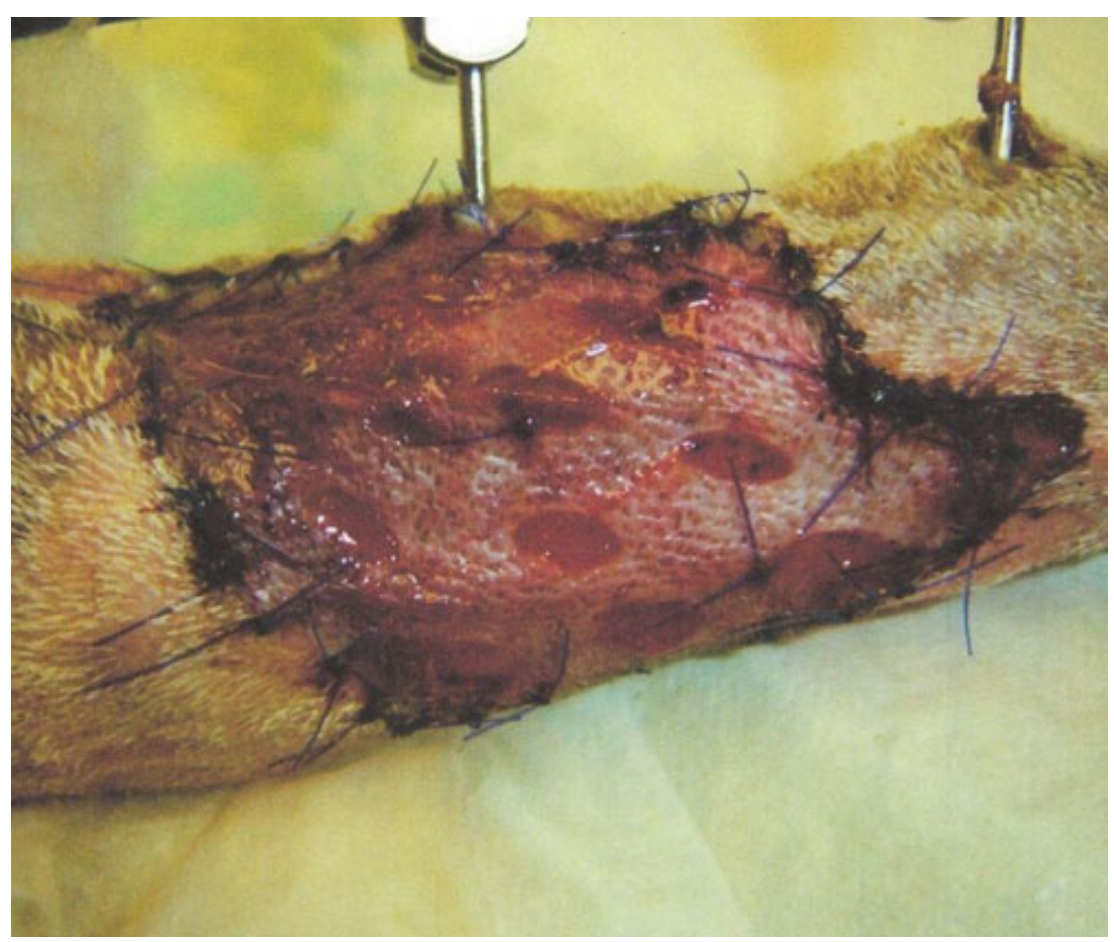

Figure 5: Day 1 post skin graft sutured in place

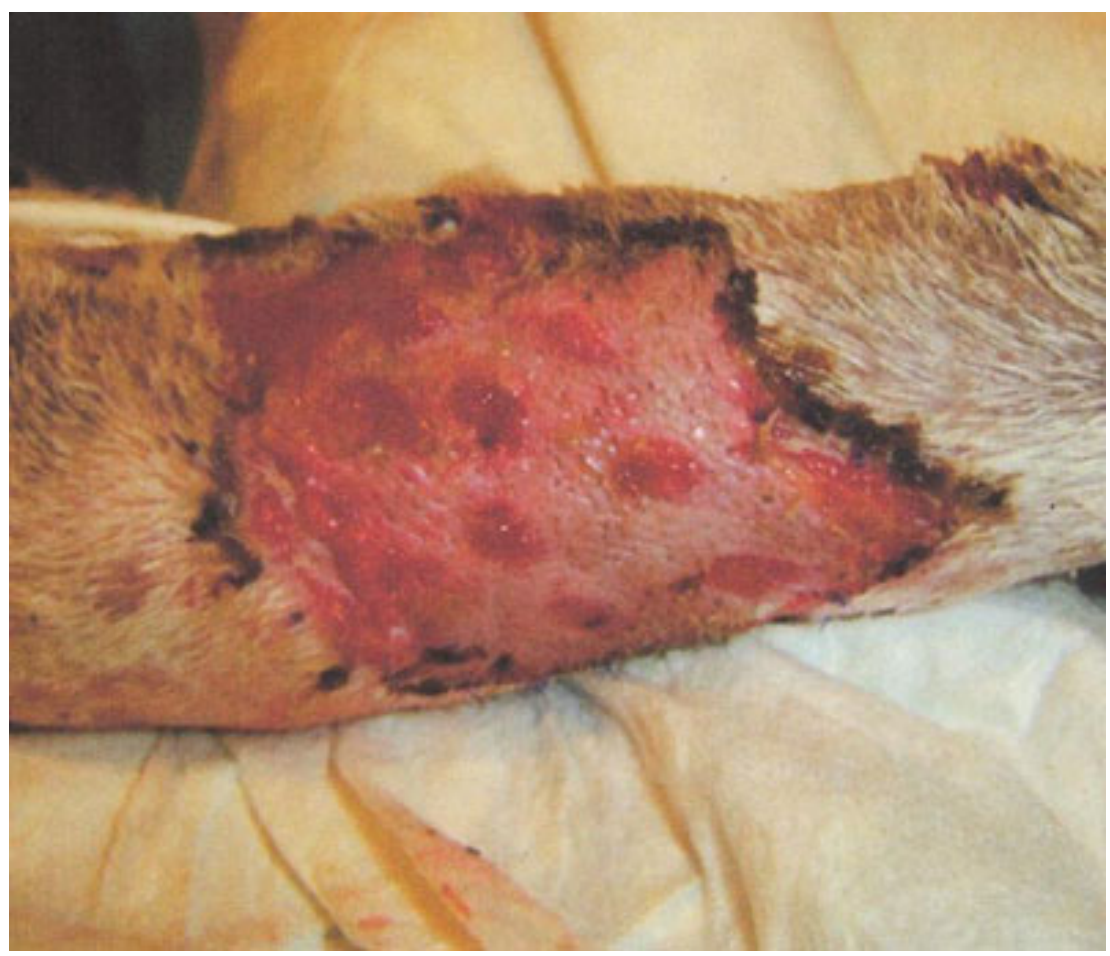

Figure 6: Day 7 post graft with no infection evident 


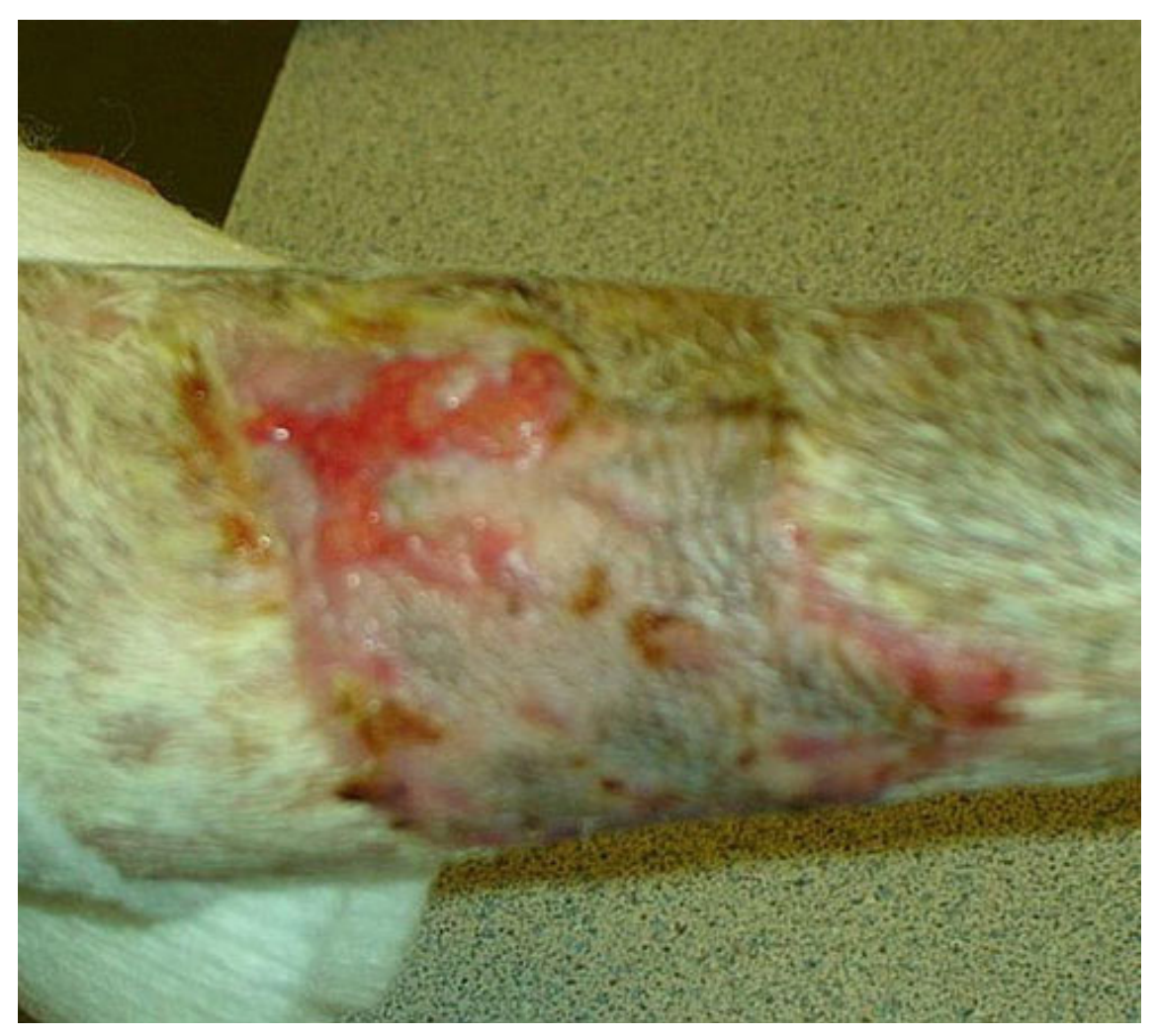

Figure 7: Day 14 post graft healing with epithelialization

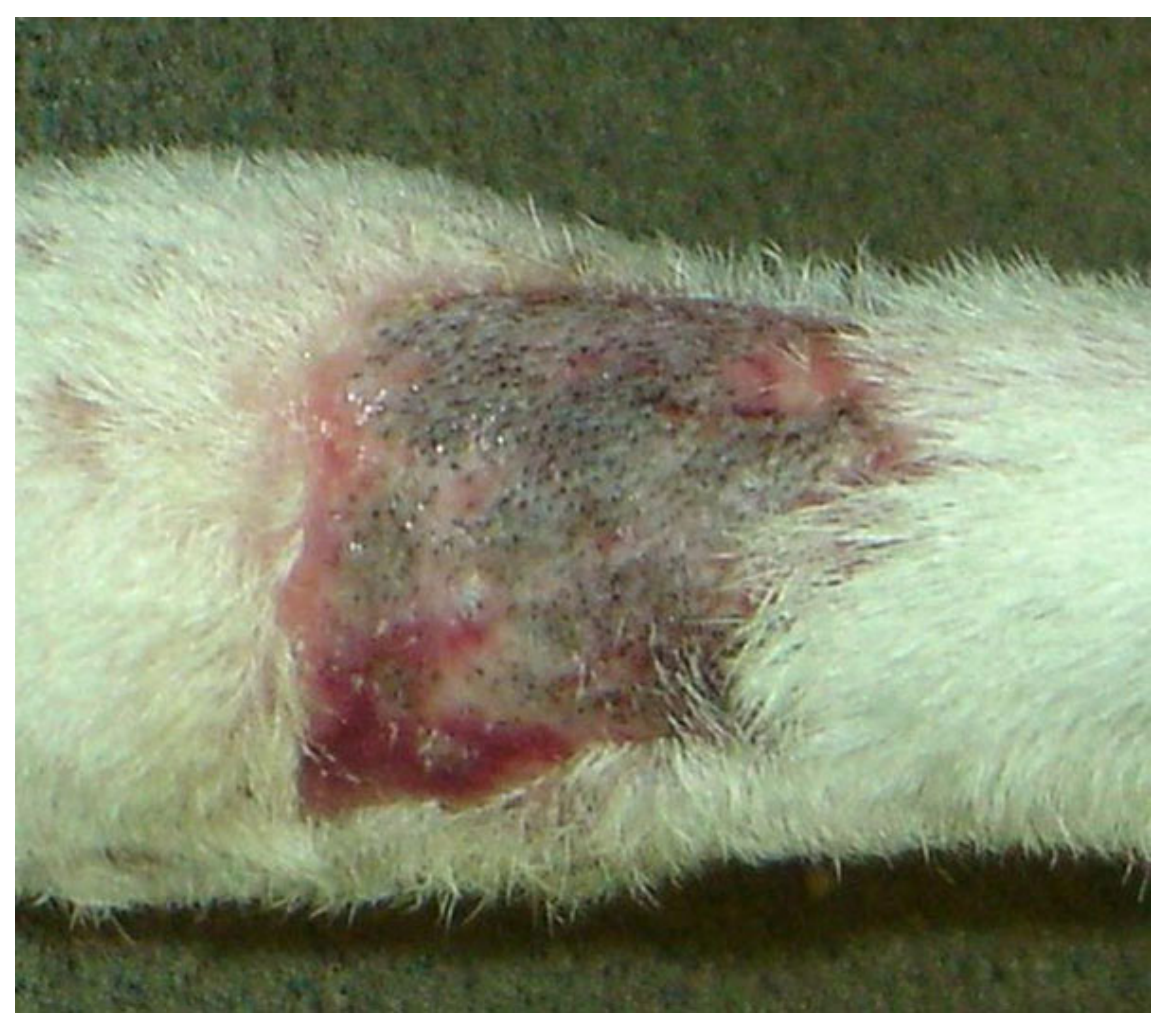

Figure 8: Day 35 post graft with some early hair re-growth 


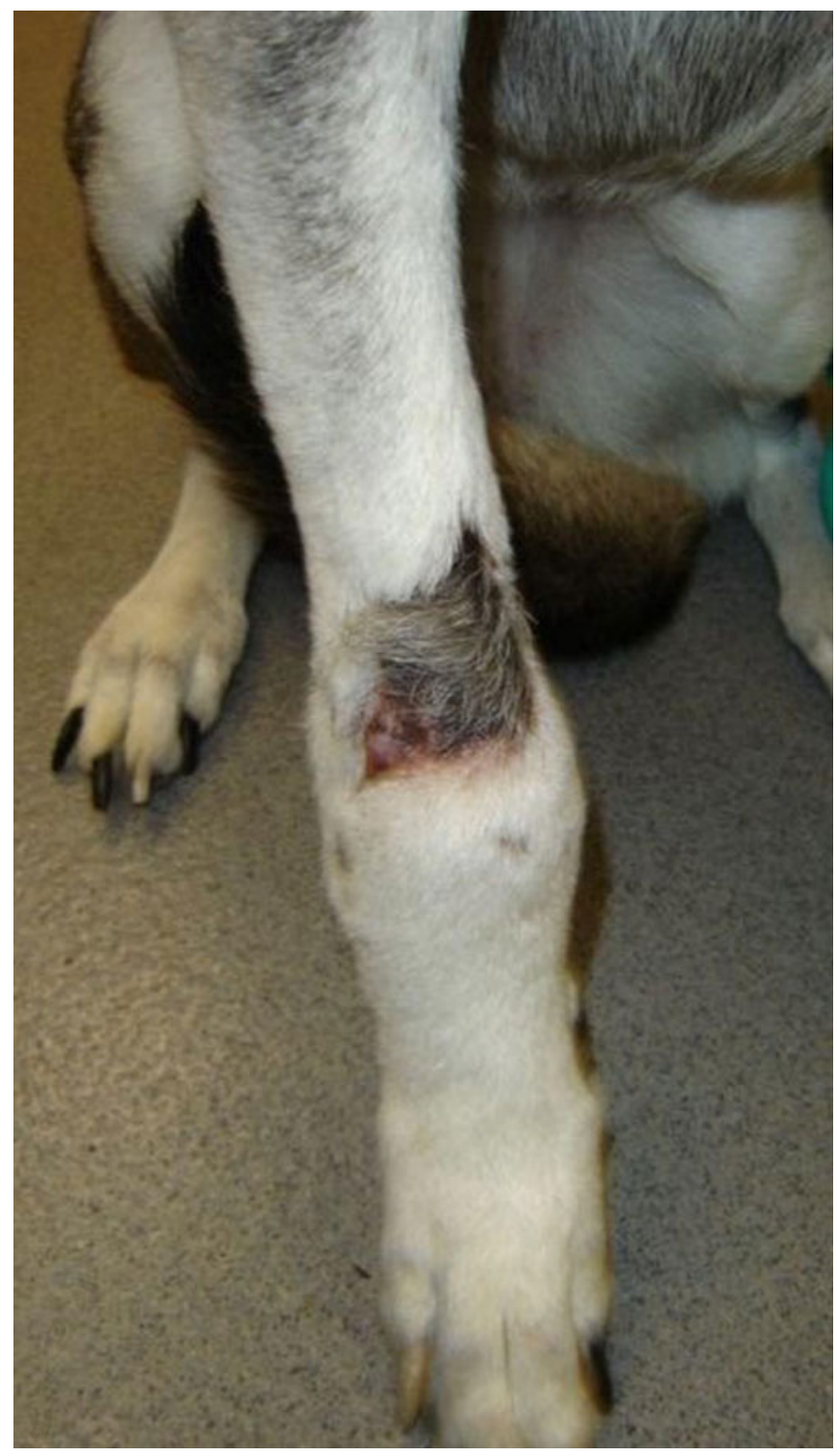

Figure 9: Day 42 post skin graft

\section{Case Study 3}

A 7 year old, female spayed, Golden Retriever was presented with right forelimb lameness. Radiographs showed a lysis of the proximal humerus with exhuberant periosteal new bone formation. Histopathology diagnosed a Grade 1 chondroblastic osteosarcoma. A right scapulohumeral forelimb amputation was elected. Eight days after the amputation was performed the dog developed a hemorrhagic purulent discharge from the middle of the incision with skin necrosis at the proximal aspect of the incision. A culture was submitted. The dog was placed on ciprofloxacin and cephalexin pending culture results. An antibiotic resistant Staphylococcus schleiferi was isolated. The organism was found resistant to both antibiotics. The dog was changed to clindamycin. Two days later (10 days after surgery) the wound had completely dehisced (Figure 10).

At this point surgical debridement of the wound was performed and treatment with chlorine dioxide solution* started. The dog received daily bandage changes that included wound flushing with saline, then sprayed with chlorine dioxide solution which was left on the wound (Figures 11 \& 12). Thirty days after the initial debridement the wound had almost completely contracted. At that point the dog was anesthetized and the wound was closed primarily and went on to completely heal without further treatment (Figure 13). 


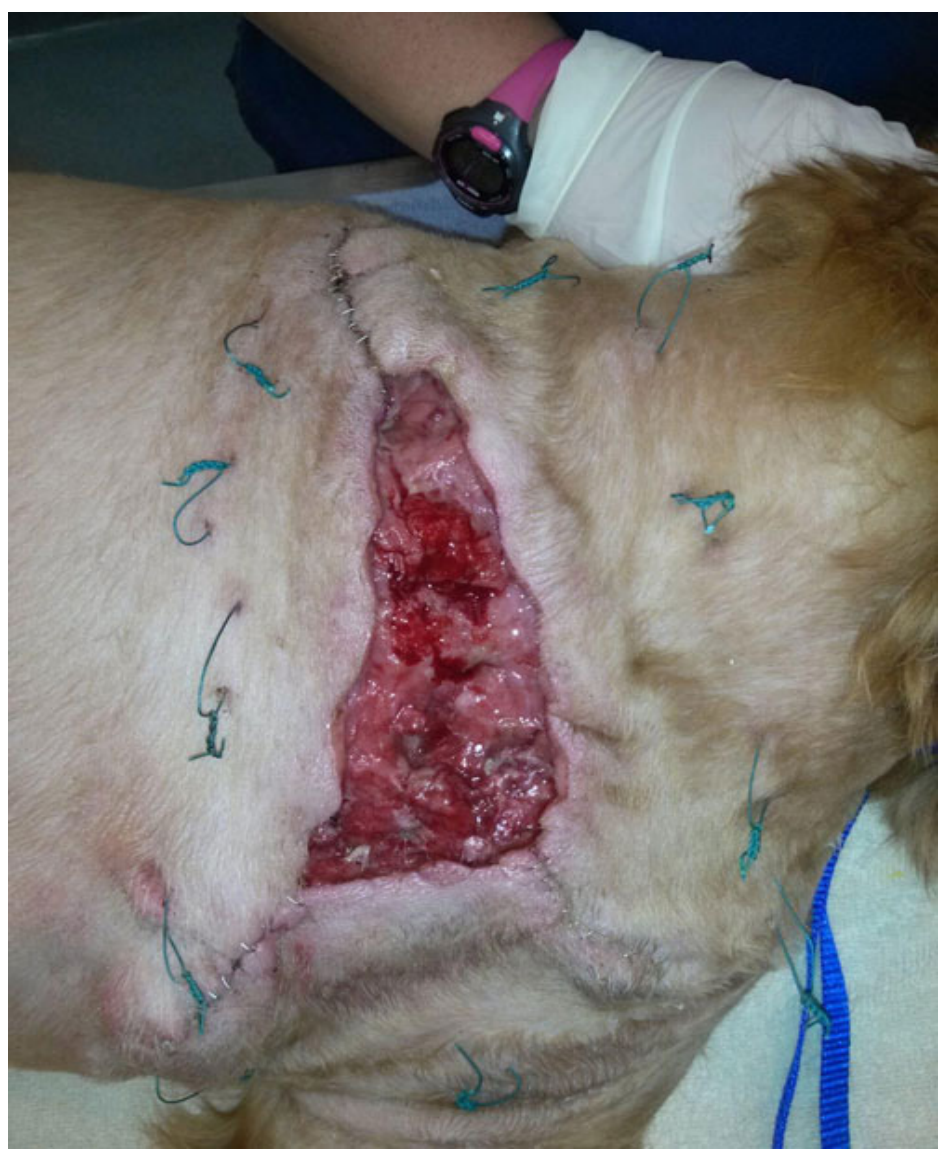

Figure 10: Open wound 2 days post debridement (12 days post initial amputation)

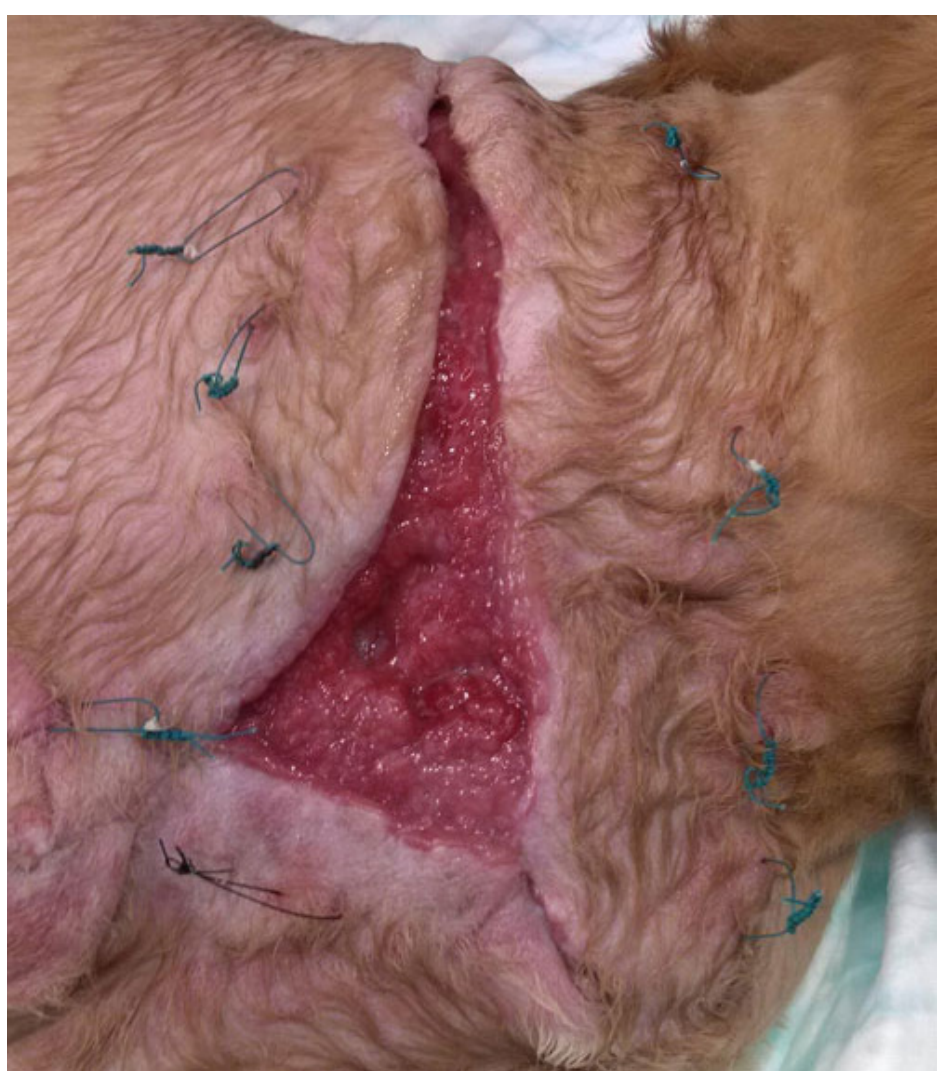

Figure 11: Wound 13 days post debridement and daily chlorine dioxide solution treatment 


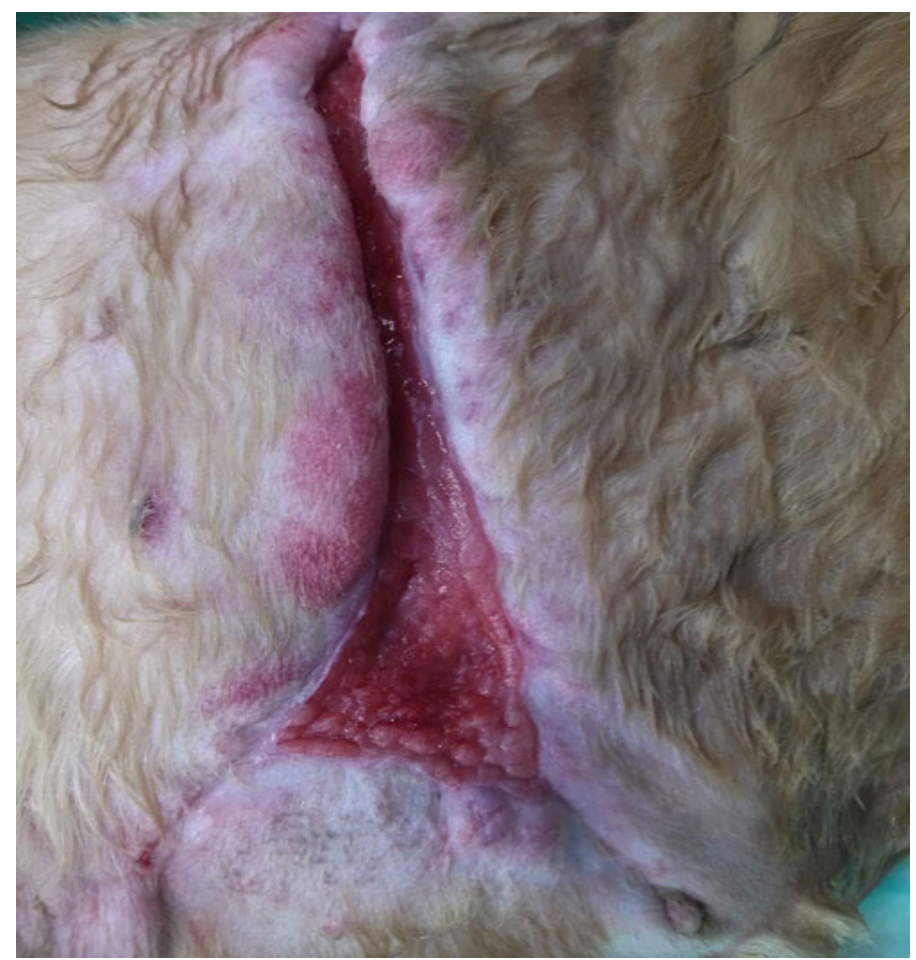

Figure 12: Wound 20 days post debridement

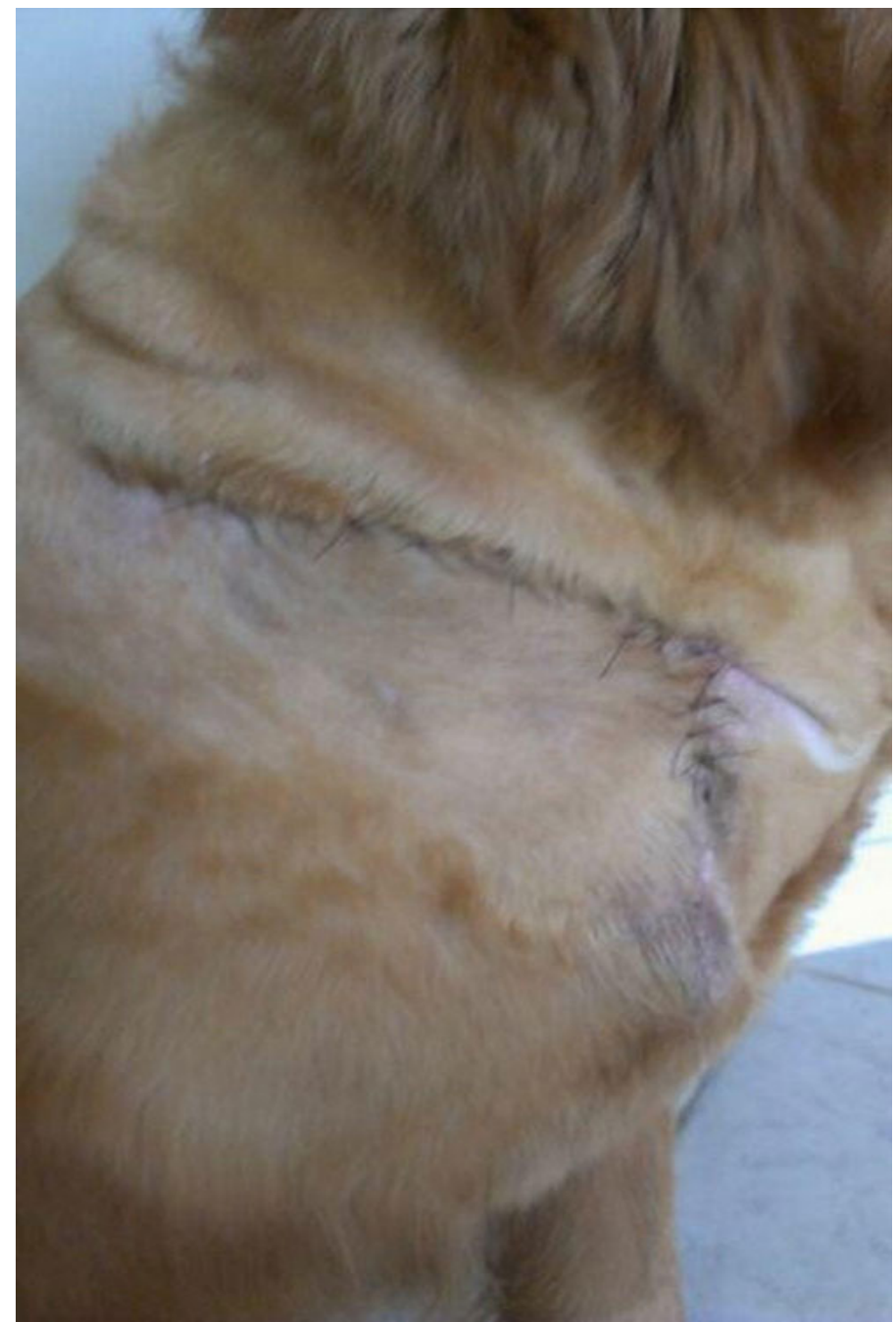

Figure 13: Wound 30 days post debridement with surgical closure 


\section{In Vitro Study 1}

In Vitro studies using some common bacterial, yeast and fungal skin pathogens isolated from clinical cases in small animal practice were tested to demonstrate the efficacy of chlorine dioxide complex solution*. The organisms included antibiotic resistant bacteria Staphylococcus aureus, Pseudomonas aeruginosa and Escherichia coli, yeast, Malassezia pachydermatis and dermatophyte fungus Microsporum canis.

A standardized suspension of each bacteria was cultured onto two trypticase soy agar plates. One untreated plate was used as a control, the other was immediately sprayed with chlorine dioxide complex solution * containing 160 ppm. Following incubation for 18 hours the plates were examined for bacterial colony growth. No growth was observed on the chlorine dioxide treated plates whereas abundant growth of bacterial colonies (Figure 14) was observed on the untreated control plate. Identical results were obtained with the cultures of Pseudomonas aeruginosa and Escherichia coli which are not shown.

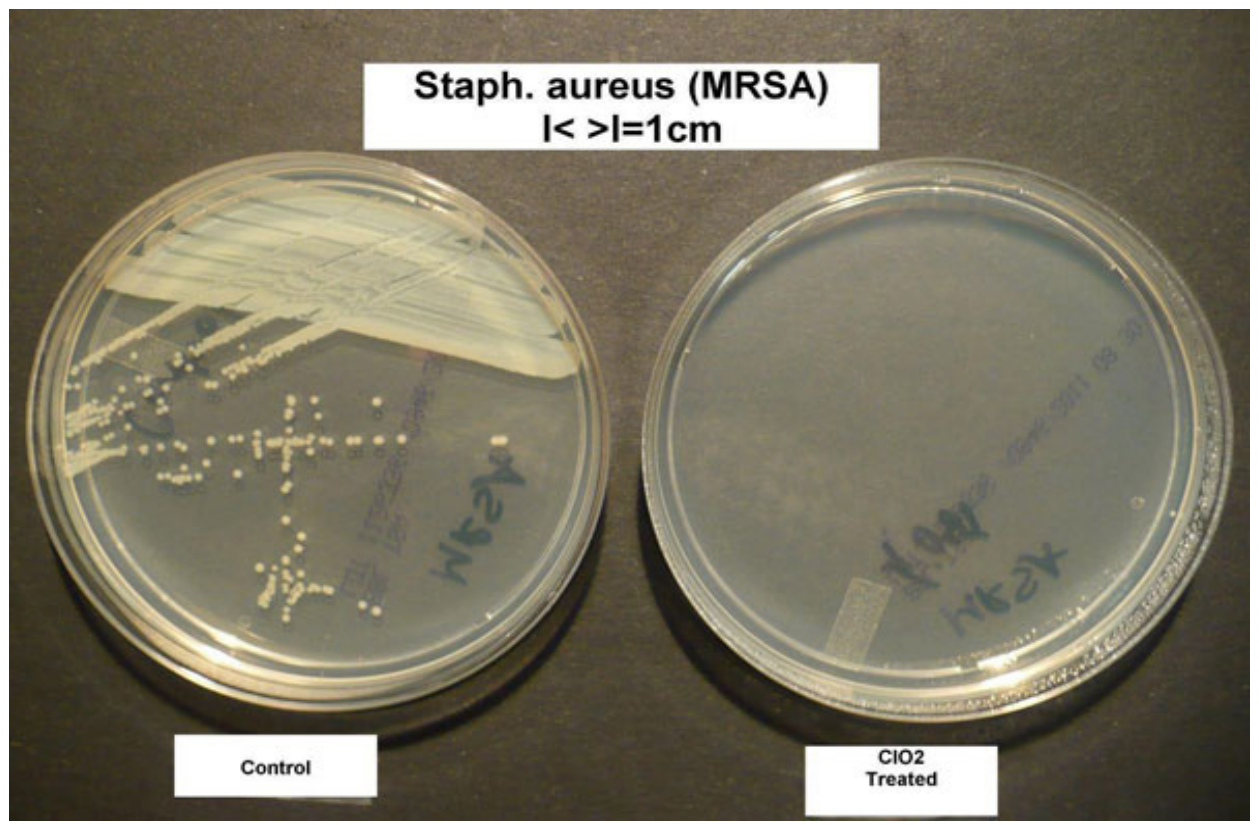

Figure 14: Staphylococcus aureus. Treated plate on right

The isolated yeast and fungal organisms were each cultured directly onto two Sabouraud Dextrose Agar plates respectively. One plate of each specimen was sprayed with the chlorine dioxide complex solution immediately and again after 24 hours. The second control plate was untreated. The plates were sealed and incubated for a total of 12 days at room temperature then inspected and photographed (Figures $15 \& 16)$.

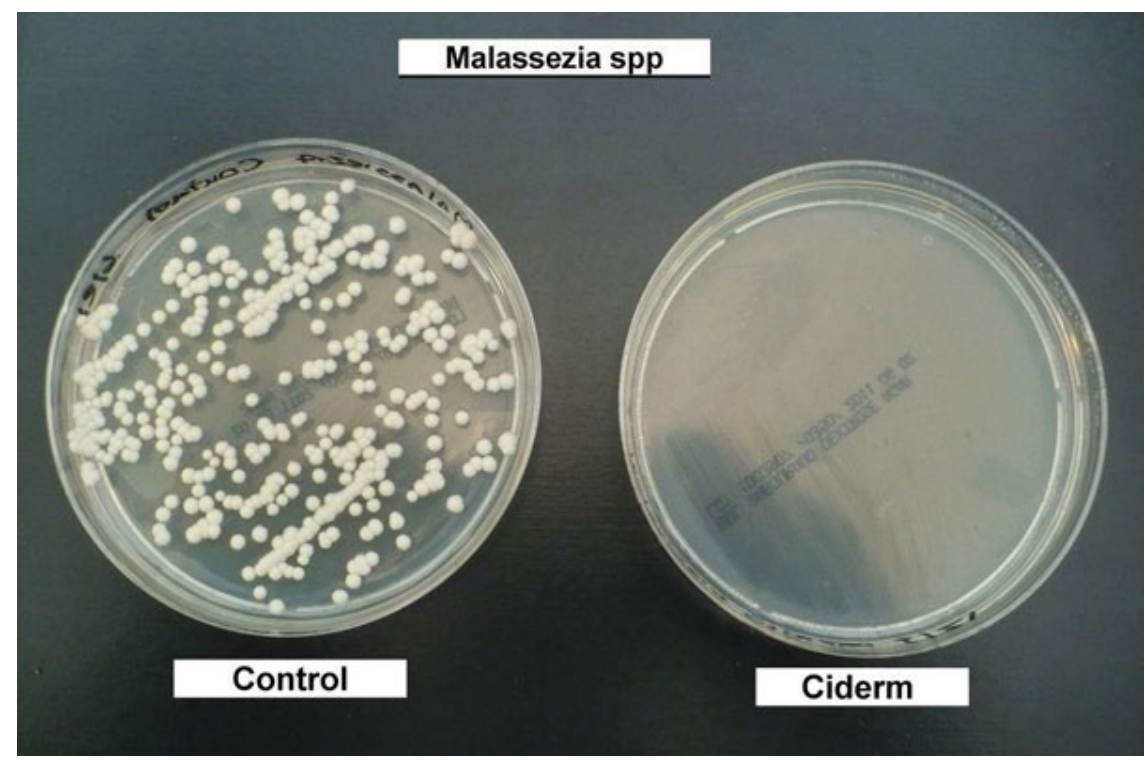

Figure 15; Malassezia spp. Treated plate on right 


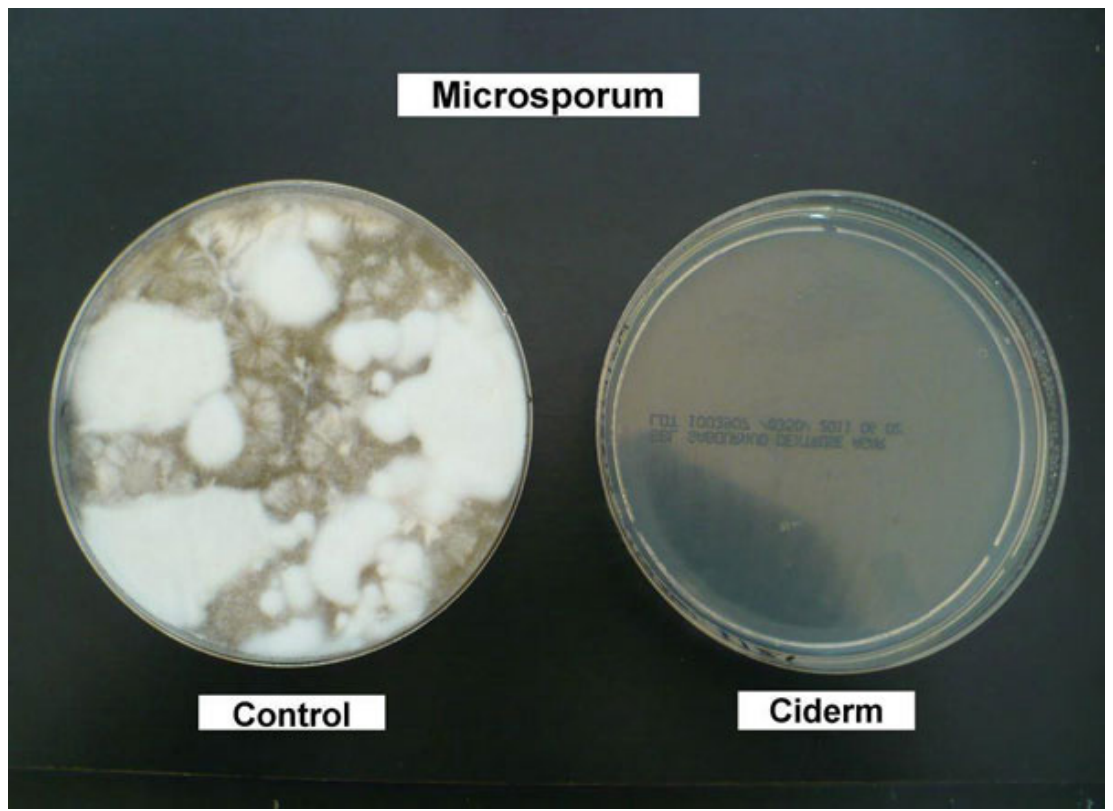

Figure 16: Microsporum spp. Treated plate on right

\section{In Vitro Study 2}

A transmission electron micrographs (TEM) taken of Pseudomonas spp bacteria demonstrated the antimicrobial effects of chlorine dioxide. Figure 17 reveals the rupture of the cell wall along with disruption of the internal organelles that occurred within 1 minute post exposure to chlorine dioxide solution at a low concentration of $10 \mathrm{ppm}$.

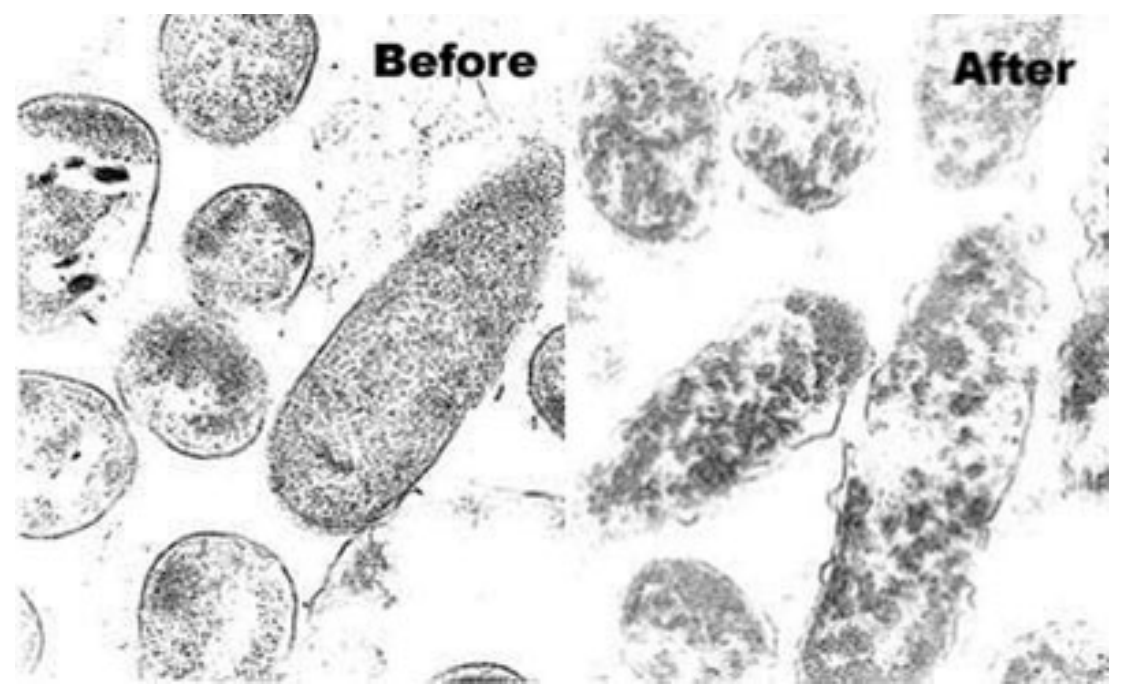

Figure 17: TEM 108,000 magnification. Pseudomonas aeruginosa organisms after one minute exposure to chlorine dioxide solution.

\section{Discussion}

The three case studies are representative of 32 cases treated successfully in a one year period using topical application of chlorine dioxide antiseptic solution ${ }^{\star}$ in the post operative management of the wounds. All three cases described, presented with complicated infected wounds. In cases 1 and 3 the wounds had not responded to conventional wound management that included surgical intervention, debridement with closure, postoperative irrigation and systemic antibiotic coverage. The wounds had dehisced and become secondarily infected. The quick response to clearing of the superficial infection using chlorine dioxide topical application and, or irrigation supports the effectiveness of this antiseptic. Systemic antibiotics were used in conjunction to prevent potential systemic spread of infection in these clinical cases.

Chlorine dioxide $\left(\mathrm{ClO}_{2}\right)$ is a gas that dissolves readily in water [1,2]. The molecule is a free radical with an unpaired electron which makes the compound particularly active as an oxidizing agent. Unlike chlorine $\left(\mathrm{Cl}_{2}\right)$, chlorine dioxide is eco-friendly. It 
does not hydrolyze in water to form hydrochloric acid but remains in solution as a dissolved gas[5]. Chlorine dioxide does not form chlorinated hydrocarbons which can be carcinogenic and irritating to skin, wounds and mucous membranes[3]. Chlorine dioxide is a strong oxidizing agent and fast acting antimicrobial agent effective at low concentration [3,4]. It is widely used as a water purifier[1,2], surface disinfectant [4] and as a topical antiseptic agent with a wide spectrum of microbicidal activity against bacteria, yeast, fungi, protozoa, and viruses $[3,6]$. The aqueous solution, at appropriate concentration, is non-irritating to the skin or wounds [3,5]. Animal studies have shown that scarring is minimized when wounds are treated with $\mathrm{ClO} 2$ liquid and gels[7]. The new proprietary formulation of chlorine dioxide as a molecular complex has proved stable in aqueous solution. This current study documents the effectiveness of this solution in managing post operative wounds, in preventing secondary infection while proving non-irritating to the dogs skin or wounds

\section{Conclusion}

The new formulation of a stabilized complex form of chlorine dioxide in an aqueous solution* at a concentration of 160 ppm provided good antisepsis in treating complicated post surgical wounds which are readily subject to secondary infections. This formula $\neg$ tion is a convenient effective antiseptic for use in conjunction with conventional wound management in clinical practice.

\section{Acknowledgement}

Howard Alliger : President Frontier Pharmaceutical Inc: Melville NY 11747

\section{References}

1. Walther HJ (1979) Masschelein, W. J. (Edt. for publication in English: Rice, R. G.): Chlorine Dioxide, Chemistry and Environmental Impact of Oxychlorine Compounds. Ann Arbor Science Publishers, Michigan, USA.

2. Aieta E, Berg JD (1986) A review of chlorine dioxide in drinking water treatment. J Am Water Works Assoc 78: 62-76.

3 Alliger H (2001) An Overall View of ClO2. Frontier Pharmaceutical Inc, Melville, New York, USA.

4. Benarde MA, Snow WB, Olivieri VP, Davidson B (1967) Kinetics and mechanism of bacterial disinfection by chlorine dioxide. Appl Microbiol 15: $257-65$.

5. Harrington R., Gates D, Romano R (1989) A review of the uses chemistry and health effects of chlorine dioxide and the chlorite ion. Chlorine Dioxide Panel of the Chemistry Manufacturers Association, Washington D.C, USA.

6. Kenyon AJ, Hamilton SG, Douglas DM (1986) Comparison of antipseudomonad activity of chlorine dioxide/chlorous acid-containing gel with commercially available antiseptics. Am J Vet Res 47: 1101-4.

7. Kenyon AJ, Hamilton SG, Douglas DM (1986) Controlled wound repair in guinea pigs, using antimicrobials that alter fibroplasia. Am J Vet Res 47: 96-101.

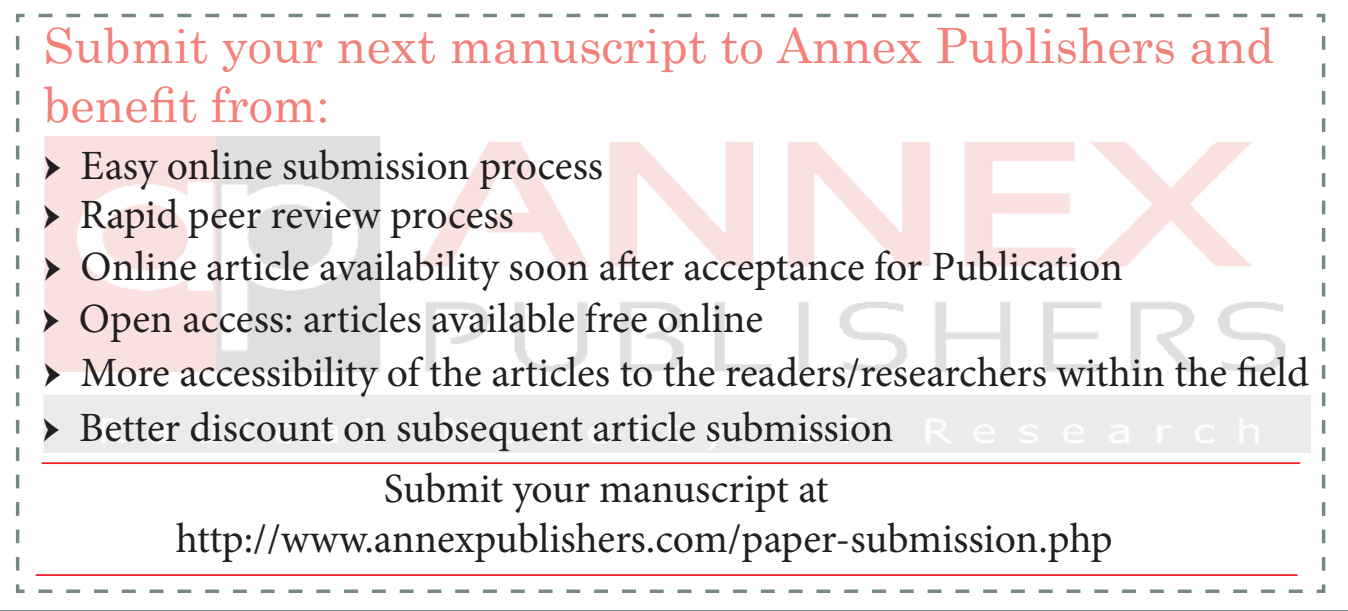

